\title{
Anti-Jamming GPS Receiver with Reduced Phase Distortions
}

\author{
Yimin D. Zhang, Senior Member, IEEE, and Moeness G. Amin, Fellow, IEEE
}

\begin{abstract}
Anti-jamming techniques are critical to maintain the integrity and functionality of GPS systems in various applications. One of the major problems with existing array-based antijamming GPS receivers is the errors introduced in the carrier phase, affecting the GPS solution. In this letter, we propose a novel anti-jamming GPS receiver structure that preserves the GPS signal phase continuity. The effectiveness of the proposed technique is verified by simulation results.
\end{abstract}

Index Terms-GPS receiver, anti-jamming, phase error, adaptive array processing, blind beamforming.

\section{INTRODUCTION}

The ever-increasing reliance on Global Positioning System (GPS) and other Global Navigation Satellite Systems (GNSS) for navigation and guidance has created a growing awareness of the need for adequate protection against both unintentional and intentional jammers. Interference caused by jamming to the GPS signals can be mitigated by using adaptive signal processing [1], [2]. In particular, adaptive array processing techniques allow exploitation of the spatial domain for improved jamming suppression and signal protection [3]-[6].

An anti-jam GPS receiver is required to be operational at "cold" start, i.e., to start with no information about its position and orientation. In this case, although the positions of GPS satellites may be found at the GPS almanac, the GPS receiver still cannot determine the steering vectors associated with the satellites until jammers are sufficiently suppressed. Blind anti-jamming array processing techniques, such as minimum variance (MV), achieve excellent jammer-suppression performance (e.g., [3], [6]). GPS coarse acquisition (C/A) signals use direct-sequence spread spectrum waveforms with 20 replicas of 1023-chip pseudo-noise (PN) codes, thus achieving a processing gain of $43.1 \mathrm{~dB}$. The high processing gain enables GPS signals to assume very low power prior to despreading and, as such, escape suppression or nulling. The sufficiently high signal level after despeading provides proper detection of signal delays for receiver positioning.

One of the major problems with the existing blind antijamming array signal processing techniques, however, is that they introduce errors in the carrier phase. These errors may cause intolerable bias in the estimated pseudo range, rendering GPS solution unacceptable for various navigation and positioning applications. In severe situations, the GPS receiver

Copyright (c) 2012 IEEE. Personal use of this material is permitted. However, permission to use this material for any other purposes must be obtained from the IEEE by sending a request to pubs-permissions@ieee.org.

The authors are with the Wireless Communications and Positioning Lab, Center for Advanced Communications, Villanova University, Villanova, PA 19085, USA (e-mails: \{yimin.zhang,moeness.amin\}@villanova.edu). may lose lock on the carrier phase and remains dysfunctional until it regains phase lock [7]. Thus, there is a pressing need to provide a high performance GPS anti-jamming solution and equip the GPS platforms with the capability to meet application precision requirements.

The objective of this letter is to develop a novel arraybased adaptive anti-jamming algorithm that blindly suppresses jammers with negligible phase distortion. The proposed array processing algorithm can effectively mitigate the GPS carrier phase distortion in jamming and non-jamming environments. The key idea behind the proposed algorithms is achieving GPS signal phase continuity by using accurate estimation of phase-rotated steering vector of the GPS signal. This is accomplished in the presence of strong jammers and high noise power and in a dynamic operational environment. The specific signal characteristics and temporal structures of GPS coarse acquisition (C/A) codes are used for improved steering vector estimation. The effectiveness of the proposed algorithms is verified by simulation results.

\section{Signal Model and Problem Statement}

\section{A. Signal Model}

Without loss of generality and for the convenience of description, we consider the reception of one GPS satellite signal. The GPS signal, along with jammers and noise, is received at each antenna of a multi-sensor array, down-converted using a phase-synchronized down-converter and digitized using an analog-to-digital converter. The signals received by an $N$ element array can be expressed, using complex baseband representations, as the following $N \times 1$ vector

$$
\mathbf{x}(t)=\mathbf{s}(t)+\mathbf{j}(t)+\mathbf{n}(t)
$$

where $\mathbf{s}(t)=\mathbf{a}_{s} s(t)$ is the GPS signal vector with $\mathbf{a}_{s}$ denoting the steering vector associated with the direction of the GPS satellite, and $s(t)$ is the GPS waveform. In addition, $\mathbf{j}(t)=\sum_{k=1}^{K} \mathbf{a}_{k} j_{k}(t)$ is the summation of $K$ jamming signals with $\mathbf{a}_{k}$ and $j_{k}(t)$ denoting the respective steering vector and waveform of the $k$ th jammer, $k=1, \ldots, K$. The elements of the additive noise vector $\mathbf{n}(t)$ are assumed to be independent and identically distributed (i.i.d.) circular symmetric complex Gaussian with zero mean and variance $\sigma_{n}^{2}$. In practice, the GPS signals have a very low power before despreading, whereas strong jammers are assumed, as the use of spreading spectrum signal equips standard GPS receiver with certain protection against weak jammers.

\section{B. Jammer Suppression}

An $N \times 1$ weight vector, denoted as $\mathbf{w}$, is applied to signal vector $\mathbf{x}(t)$. If the steering vector of the GPS satellite 
signal, $\mathbf{a}_{s}$, is known, we could apply the minimum variance distortionless response (MVDR) algorithm described by [8]:

$$
\begin{aligned}
& \min _{\mathbf{w}} P_{\text {out }}=\mathbf{w}^{H} \mathrm{E}\left[\mathbf{x}(t) \mathbf{x}^{H}(t)\right] \mathbf{w}=\mathbf{w}^{H} \mathbf{R} \mathbf{w} \\
& \text { subject to } \mathbf{w}^{H} \mathbf{a}_{s}=1,
\end{aligned}
$$

where $(\cdot)^{H}$ denotes conjugate transpose, and $\mathrm{E}[$.$] denotes$ statistical expectation. The solution corresponding to the above problem is given by

$$
\mathbf{w}=\frac{\mathbf{R}^{-1} \mathbf{a}_{s}}{\mathbf{a}_{s}^{H} \mathbf{R}^{-1} \mathbf{a}_{s}},
$$

where $(\cdot)^{-1}$ denotes matrix inversion. It is noteworthy hat the above weight vector does not cause any phase distortion to the received GPS signal as it can be readily shown that $\mathbf{w}^{H} \mathbf{s}(t)=$ $\mathbf{w}^{H} \mathbf{a}_{s} s(t)=s(t)$. As described earlier, however, the steering vector of the GPS signal is often unavailable in practice at "cold" start. As such, an anti-jamming GPS receiver is required to suppress jammers in the absence of such knowledge. One possible solution is to use the MV algorithm, in which the weight vector is determined as

$$
\mathbf{w}=\frac{\mathbf{R}^{-1} \mathbf{e}_{1}}{\mathbf{e}_{1}^{H} \mathbf{R}^{-1} \mathbf{e}_{1}}
$$

where $\mathbf{e}_{1}=[1,0, \ldots, 0]^{T}$ is a steering vector which, by itself without anti-jamming operation, corresponds to an omnidirectional array pattern, and $(\cdot)^{T}$ denotes transpose. In essence, instead of using the steering vector corresponding to the GPS signal $\mathbf{a}_{s}$ as in the MVDR algorithm, the MV algorithm uses $\mathbf{e}_{1}$ which does not make a preference for any direction. As a result, an MV beamformer tends to suppress any strong directional signal arrivals such that the overall array output becomes spatially white.

In practice, the weight vector $\mathbf{w}$ is periodically updated, depending on the operation environment. Thus, the weight vector at time instant $t$ is obtained as

$$
\mathbf{w}_{t}=\frac{\mathbf{R}_{t}^{-1} \mathbf{e}_{1}}{\mathbf{e}_{1}^{H} \mathbf{R}_{t}^{-1} \mathbf{e}_{1}},
$$

where $\mathbf{R}_{t}$ is an estimate of the covariance at time instant $t$, which is usually obtained by using a block of data immediately before $t$. Because of its weak power prior to despreading, the GPS signal makes negligible contributions to $\mathbf{R}_{t}$ and thus will go unnoticed and transparent to the MV algorithm. However, the $\mathrm{MV}$ algorithm yields phase change because in

$$
\mathbf{w}_{t}^{H} \mathbf{s}(t)=\mathbf{w}_{t}^{H} \mathbf{a}_{s} s(t)=\frac{\mathbf{e}_{1}^{T} \mathbf{R}_{t}^{-1} \mathbf{a}_{s}}{\mathbf{e}_{1}^{H} \mathbf{R}_{t}^{-1} \mathbf{e}_{1}} s(t),
$$

the term $\mathbf{w}_{t}^{H} \mathbf{a}_{s}$ in general takes a complex value with its phase depending on $\mathbf{R}_{t}$. That is, when jammers appear or disappear from the scene, the corresponding weight vectors may yield significant phase discontinuity of the output GPS signal.

\section{Existing Technique for Phase Compensation}

To overcome the above problem, a phase compensation method was developed in [8] by adjusting the numericallycontrolled oscillator (NCO) based on the phase difference between the current and the previous time samples. In particular, it computes the effective phase change in the signal which is given by

$$
\sum_{n=1}^{N} w_{n, t+1}^{*} m_{n} e^{\imath \theta_{n}}-\sum_{n=1}^{N} w_{n, t}^{*} m_{n} e^{\imath \theta_{n}},
$$

where $\imath=\sqrt{-1}, w_{n, t}$ is the $n$th element of the weight vector at time instant $t$, and $m_{n} e^{\imath \theta_{n}}$ represents the magnitude and phase of the response of the $n$th antenna to the GPS signal and is assumed to be constant over two consecutive weight updates. Because $m_{n} e^{\imath \theta_{n}}$ is in fact the $n$th element of the steering vector of the GPS signal, i.e., $\mathbf{a}_{s}$, which is unavailable at "cold start" as described previously, the method developed in [8] computes the phase difference between the two weight update periods as

$$
\Delta \zeta=\angle\left[\sum_{n=1}^{N} w_{n, t+1}^{*}\right]-\angle\left[\sum_{n=1}^{N} w_{n, t}^{*}\right],
$$

where $\angle$ denotes the phase of a complex scalar.

In the approach developed in [8], the phase difference computed in equation (8) is conveyed to the carrier loop NCO across the control path. As such, the NCO utilizes the information $(\Delta \zeta)$ and tunes the carrier $\mathrm{NCO}$ to generate a local "matched" copy of the incoming signal that has been affected by a phase change imposed by the adaptive processing mechanism.

The concept proposed in [8], summarized above, is important and based on the idea of phase compensation after the jammers have been suppressed with null-steering techniques. However, it is evident from the comparison of equations (7) and (8) that $\Delta \zeta$ correctly represents the phase difference of the GPS signal only when the steering vector elements of the GPS, corresponding to different antennas, have identical phase. For example, for a planar array that consists of non-colinear elements, the result expressed in equation (8) represents the correct phase change only when the GPS signal arrives perpendicular to the array plane (elevation angle $=0^{\circ}$ ). Also, the necessity of conveying the phase error to the NCO requires anti-jamming processing to be coupled with other parts of the GPS receiver, making the system more complicated.

\section{Proposed TeChNiQUe}

\section{A. Outline of the Proposed Technique}

We develop a novel technique that correctly tracks and compensates for the phase change in different jamming environments. Similar to [8], our technique is based on nullsteering, e.g., the MV algorithm, for jammer suppression. As such, it requires no a priori information of the direction or steering vector of the GPS signal, or the array structure and manifold. The key novelty of the proposed algorithm is that it estimates a phase rotated steering vector which, in turn, is used to obtain the phase change of the GPS signal output from the previous weight update. This phase change is then equalized, preserving the phase continuity between two consecutive weight update periods. The block diagram of the proposed algorithm is depicted in Fig. 1.

The proposed anti-jamming GPS algorithm provides stable GPS signal phase output and is robust to the imperfect knowledge of the operational environment (e.g., calibration errors). 
In essence, it provides precise estimation and compensation of the GPS phase change without the need of involving the NCO or a similar device. The proposed algorithm is presented in this section, whereas its implementation based on the sample matrix inversion (SMI) technique is described in the next section.

Because it is difficult to precisely estimate $\mathbf{a}_{s}$ without phase uncertainty in a blind manner, we first develop a technique that provides an accurate estimation of a steering vector of the GPS signal, up to a complex scalar, denoted as $\tilde{\mathbf{a}}_{s}=\mathbf{a}_{s} \alpha e^{\imath \vartheta}$, where $\alpha$ is a real scalar, and $\vartheta$ is an unknown phase rotation. The data is segmented into equal-length blocks, and the array weight vector is updated in each block according to the environment changes. Assume that the steering vector of a GPS signal does not change over two consecutive weight update periods. This is a reasonable assumption since the weight vector is usually updated over a short time period, e.g., every few milliseconds. Notice, however, that the operational environment may change over that time period, i.e., a jammer may appear or disappear from the scene. Assume that the weights are updated every $D$ samples. Then, the phase of $\mathbf{w}_{t+D}$ must be adjusted so that the following relationship holds

$$
\angle \mathbf{w}_{t+D}^{H} \tilde{\mathbf{a}}_{s}-\angle \mathbf{w}_{t}^{H} \tilde{\mathbf{a}}_{s}=0 .
$$

This relationship ensures phase continuity of the output GPS signal between two consecutive weight vectors $\mathbf{w}_{t}$ and $\mathbf{w}_{t+D}$. Note that this is equivalent to $\angle \mathbf{w}_{t+D}^{H} \mathbf{a}_{s}-\angle \mathbf{w}_{t}^{H} \mathbf{a}_{s}=0$, because

$$
\begin{aligned}
\angle \mathbf{w}_{t+D}^{H} \tilde{\mathbf{a}}_{s}-\angle \mathbf{w}_{t}^{H} \tilde{\mathbf{a}}_{s} & =\left(\angle \mathbf{w}_{t+D}^{H} \alpha \mathbf{a}_{s}+\vartheta\right)-\left(\angle \mathbf{w}_{t}^{H} \alpha \mathbf{a}_{s}+\vartheta\right) \\
& =\angle \mathbf{w}_{t+D}^{H} \mathbf{a}_{s}-\angle \mathbf{w}_{t}^{H} \mathbf{a}_{s} .
\end{aligned}
$$

Therefore, phase continuity, described in (9), can be enforced once a good estimate of $\tilde{\mathbf{a}}_{s}$ is obtained. Below, we propose a technique that obtains a phase-rotated estimate, $\tilde{\mathbf{a}}_{s}$, of the steering vector of the GPS signal.

\section{B. Estimation of GPS Steering Vector Up to a Complex Scalar}

The unnormalized result of the MV algorithm (without using the $\mathbf{e}_{1}^{H} \mathbf{R}^{-1} \mathbf{e}_{1}$ term) can be expressed as

$$
\begin{aligned}
z_{1}(t) & =\mathbf{e}_{1}^{H} \mathbf{R}^{-1} \mathbf{x}(t)=\mathbf{e}_{1}^{H} \mathbf{R}^{-1}[\mathbf{s}(t)+\mathbf{j}(t)+\mathbf{n}(t)] \\
& \approx \mathbf{e}_{1}^{H} \mathbf{R}^{-1}[\mathbf{s}(t)+\mathbf{n}(t)],
\end{aligned}
$$

where the last approximation stems from the fact that, as previously discussed, the strong jammers are sufficiently suppressed due to the spatial whitening capability of the MV algorithm and become negligible after the MV algorithm is applied. Alternatively, instead of $\mathbf{e}_{1}=[1,0, \ldots, 0]^{T}$, we can similarly use $\mathbf{e}_{2}=[0,1,0, \ldots, 0]^{T}, \ldots, \mathbf{e}_{N}=[0,0, \ldots, 0,1]^{T}$ to obtain $z_{i}(t)=\mathbf{e}_{i}^{H} \mathbf{R}^{-1} \mathbf{x}(t) \approx \mathbf{e}_{i}^{H} \mathbf{R}^{-1}[\mathbf{s}(t)+\mathbf{n}(t)]$ for $i=1, \ldots, N$. By obtaining all the $N$ outputs and stacking the result together, we obtain a spatially whitened data vector

$$
\begin{aligned}
\mathbf{z}(t) & =\left[z_{1}(t), z_{2}(t), \ldots, z_{N}(t)\right]^{T}=\mathbf{R}^{-1} \mathbf{x}(t) \\
& \approx \mathbf{R}^{-1}[\mathbf{s}(t)+\mathbf{n}(t)]=\mathbf{R}^{-1}\left[\mathbf{a}_{s} s(t)+\mathbf{n}(t)\right],
\end{aligned}
$$

where we used the fact that $\left[\mathbf{e}_{1}, \ldots, \mathbf{e}_{N}\right]=\mathbf{I}_{N}$ with $\mathbf{I}_{N}$ denoting the $N \times N$ identity matrix. The first step is to estimate a phase-rotated version of $\mathbf{R}^{-1} \mathbf{a}_{s}$. While $\mathbf{z}(t)$ contains this component as depicted in (12), the low SNR does not warrant reliable estimation of a phase rotated version of $\mathbf{R}^{-1} \mathbf{a}_{s}$ from $\mathrm{E}\left[\mathbf{z}(t) \mathbf{z}^{H}(t)\right]$. Instead, we utilize the self-coherence characteristics of the GPS C/A code that replicates itself 20 times in each symbol to achieve GPS signal enhancement. The self-coherence characteristics have been successfully used to develop a blind anti-jamming algorithm for GPS receivers [6], [9]. That is, provided that we operate within the same symbol, the GPS signal is identical to its own delayed signal by $k P$ chips, where $k$ is an integer, and $P=1023$ chips is the length of spreading code in each repetition. Without the knowledge of the starting point of a symbol, it is certain that $20-k$ out of every 20 pairs have the same sign, whereas the other one may, with equal probability, have the same sign or a different sign.

Because the self-coherence property is only assumed by the GPS signal and not by the noise and, in most cases, the jammer residues, we have [9]

$$
\begin{aligned}
& \mathrm{E}\left[\mathbf{z}(t) \mathbf{z}^{H}(t+k P)\right] \\
& \approx \mathbf{R}^{-1} \mathrm{E}\left[(\mathbf{s}(t)+\mathbf{n}(t))(\mathbf{s}(t+k P)+\mathbf{n}(t+k P))^{H}\right] \mathbf{R}^{-1} \\
& =\mathbf{R}^{-1} \mathrm{E}\left[\mathbf{s}(t) \mathbf{s}^{H}(t+k P)\right] \mathbf{R}^{-1}=\left(1-\frac{k}{20}\right) \mathbf{R}^{-1} \mathbf{R}_{s} \mathbf{R}^{-1}
\end{aligned}
$$

where $\mathbf{R}_{s}=\mathrm{E}\left[\mathbf{s}(t) \mathbf{s}^{H}(t)\right]=\mathrm{E}\left[|s(t)|^{2}\right] \mathbf{a}_{s} \mathbf{a}_{s}^{H}$ is the covariance matrix of the GPS signal vector. As such, the dominant eigenvector of $\mathrm{E}\left[\mathbf{z}(t) \mathbf{z}^{H}(t+k P)\right]$, denoted as $\mathbf{v}_{s}=\mathbf{R}^{-1} \tilde{\mathbf{a}}_{s}$, is proportional (up to a complex scalar) to $\mathbf{R}^{-1} \mathbf{a}_{s}$.

In the second step, we obtain a scaled and phase rotated copy of the steering vector of the GPS signal, $\tilde{\mathbf{a}}_{s}=\mathbf{a}_{s} \alpha e^{j \vartheta}$, by multiplying $\mathbf{R}$ with the estimated $\mathbf{v}_{s}=\mathbf{R}^{-1} \tilde{\mathbf{a}}_{s}$, i.e.,

$$
\tilde{\mathbf{a}}_{s}=\mathbf{R} \mathbf{v}_{s} .
$$

Once $\tilde{\mathbf{a}}_{s}$ is estimated, the phase continuity of the output GPS signal can be maintained by enforcing equation (9) in each iteration. Note that when the operational environment changes, it is important to use the estimated steering vector obtained in the less jammed environment in equation (9) to minimize the effect of jammer on the output GPS signal phase (this can easily be done by monitoring the overall signal power).

\section{SMI-BASED IMPLEMENTATIONS}

For the convenience of explanation, we implement the proposed algorithm based on the SMI approach, while other types of implementations remain possible. The weights are updated every $D$ samples. As such, we estimate the covariance matrix based on a block of data with length $L$, where $L$ is not necessarily related to the symbol length or the rate of weight updates. Rather, it is chosen based on the performance requirement, channel dynamics, and the complexity constraint. In practice, $L$ is preferred to be equal to or larger than $D$.

The proposed technique, as described in the previous section, is implemented using SMI as follows:

1) At time instant $t+D$, the covariance matrix is estimated using $L$ samples of data sets as $\hat{\mathbf{R}}_{t+D}=$ $\frac{1}{L} \sum_{\tau=t+D-L+1}^{t+D} \mathbf{x}(\tau) \mathbf{x}^{H}(\tau)$.

2) Compute spatially whitened data vector $\mathbf{z}(\tau)=$ $\hat{\mathbf{R}}_{t+D}^{-1} \mathbf{x}(\tau), \quad \tau=t+D-L+1, \ldots, t+D$, 
and obtain the dominant eigenvector of matrix $\frac{1}{L-P} \sum_{\tau=t+D-L+1}^{t+D-P} \mathbf{z}(\tau) \mathbf{z}^{H}(\tau+P) \triangleq \mathbf{v}_{s}$.

3) Compute the phase-rotated steering vector as $\tilde{\mathbf{a}}_{s, t+D}=$ $\hat{\mathbf{R}}_{t+D} \mathbf{v}_{s}$.

4) Compute $P_{t+D}=(1 / N) \operatorname{tr}\left(\hat{\mathbf{R}}_{t+D}\right)$ as the total power indicator, where $\operatorname{tr}(\cdot)$ denote the trace of a matrix. Choose $\tilde{\mathbf{a}}_{s}=\tilde{\mathbf{a}}_{s, t+D}$ if $P_{t+D} \leq P_{t}$, and $\tilde{\mathbf{a}}_{s}=\tilde{\mathbf{a}}_{s, t}$ if $P_{t+D}>P_{t}$, where $P_{t}$ and $\tilde{\mathbf{a}}_{s, t}$ are the total power indicator and the estimate of the phase rotated steering vector obtained at time instant $t$.

5) Compute the interim MVDR weight vector as $\hat{\mathbf{w}}_{t+D}^{\prime}=$ $\left[\tilde{\mathbf{a}}_{s, t+D}^{H} \hat{\mathbf{R}}_{t+D}^{-1} \tilde{\mathbf{a}}_{s, t+D}\right]^{-1} \hat{\mathbf{R}}_{t+D}^{-1} \tilde{\mathbf{a}}_{s, t+D}$, and calculate the phase difference $\epsilon=\angle \hat{\mathbf{w}}_{t+D}^{\prime H} \tilde{\mathbf{a}}_{s}-\angle \hat{\mathbf{w}}_{t}^{\prime H} \tilde{\mathbf{a}}_{s}$.

6) The weight vector at time instant $t+D$ is determined as $\hat{\mathbf{w}}_{t+D}=\hat{\mathbf{w}}_{t+D}^{\prime} e^{\imath \epsilon}$, which is used to compute the $D$ data samples as $y(\tau)=\hat{\mathbf{w}}_{t+D}^{H} \mathbf{x}(\tau), \tau=t+1, \ldots, t+D$.

\section{Simulation Results}

The parameters used to generate the simulation results are listed in Table 1. For comparison, the scenario is similar to [8] with minor modifications that demonstrate the rapid response of the proposed technique. The GPS starts operation at $t=0.5$ sec. A continuous wave $(\mathrm{CW})$ interferer and a broadband (BB) interferer are injected into the scenario at two different time instants, respectively at $t=1.0 \mathrm{sec}$. and $1.3 \mathrm{sec}$. The interelement spacing of the array is chosen to be 0.4 wavelength so that the antennas can fit into a 5-inch diameter platform. The waveforms are sampled once per chip, and the block data length of $L$ corresponds to a symbol period $(20 \mathrm{~ms})$. In addition to the situations where new jammers appear, we also considered the situations where existing jammers disappear from the scene (the $\mathrm{CW}$ and $\mathrm{BB}$ interferers disappear at $t$ $=1.5 \mathrm{sec}$. and $1.8 \mathrm{sec}$., respectively). The weights are updated every 5 milliseconds.

Fig. 2 depicts simulation results of the phase of the output GPS signal. The phase performance is compared with the MV algorithm without using the proposed phase compensation technique. In the simulated scenario, the maximum phase change of the proposed algorithm during the environment transition is $1.0^{\circ}$, whereas for the MV algorithm without phase compensation, the phase difference is $35.2^{\circ}$. For comparison, we also included the results that are obtained by setting $\Delta \zeta$ in (8) to 0 in each weight update step, rendering the maximum phase difference during environment transition to be $126.8^{\circ}$. Monte Carlo trials for 50 different noise realizations yield an average of the maximum phase change to be $0.7^{\circ}$ for the proposed technique, $35.9^{\circ}$ for the $\mathrm{MV}$, and $127.1^{\circ}$ for the results obtained through (8).

\section{CONCLUSION}

We have developed a novel array-based anti-jamming GPS receiver that enables effective jammer suppression with negligible phase distortion. We showed that a scaled and phase rotated steering vector of the GPS can be used to preserve phase continuity of the GPS signals in a changing jamming environment. A technique that provides a robust estimate of such steering vector was developed.

\section{REFERENCES}

[1] Y. Zhang, M. G. Amin, and A. R. Lindsey, "Anti-jamming GPS receivers based on bilinear signal distributions," in Proc. IEEE Military Commun. Conf., Vienna, VA, Oct. 2001.

[2] M. G. Amin and Y. Zhang, "Interference suppression in spread spectrum communication systems," in J. G. Proakis (ed.), The Wiley Encyclopedia of Telecommunications, John Wiley, 2002.

[3] R. Fante and J. J. Vaccaro, "Wideband cancellation of interference in a GPS receive array," IEEE Trans. Aerospace and Electronic Systems, vol. 36, no. 2, pp. 549-564, Apr. 2000.

[4] Y. Zhang and M. G. Amin, "Array processing for nonstationary interference suppression in DS/SS communications using subspace projection techniques," IEEE Trans. Signal Proc., vol. 49, no. 12 , Dec. 2001.

[5] M. G. Amin, L. Zhao, and A. R. Lindesy, "Subspace array processing for the suppression of FM jamming in GPS receivers," IEEE Trans. Aerospace and Electronic Systems, vol. 40, no. 1, pp. 80-92, Jan. 2004.

[6] W. Sun and M. G. Amin, "A self-coherence anti-jamming GPS receiver," IEEE Trans. Signal Proc., vol. 53, no. 10, pp. 39103915, Oct. 2005.

[7] B. Parkinson and J. Spilker (Eds.), Global Positioning System: Theory and Applications. AIAA, 1996.

[8] S. K. Kalyanaraman and M. S. Braasch, "GPS adaptive array phase compensation using a software radio architecture," Navigation: J. ION, vol. 57, no. 1, pp. 53-68, Spring 2010.

[9] M. G. Amin, "A self-coherence based anti-jam GPS receiver," presentation at IEEE Rhode Island Section, March 2005. http://www.ieee.li/pdf/viewgraphs/gps_anti_jam.pdf.

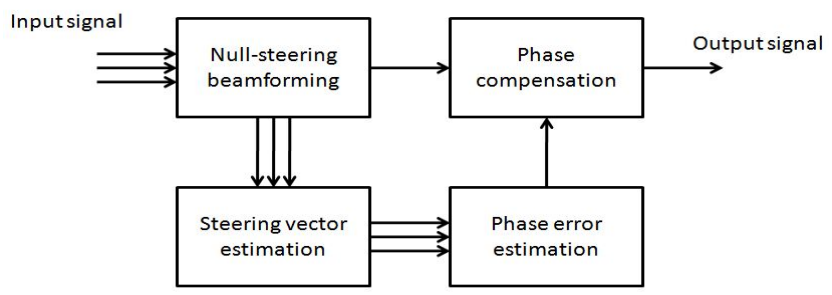

Fig. 1. Block diagram of the proposed algorithm.

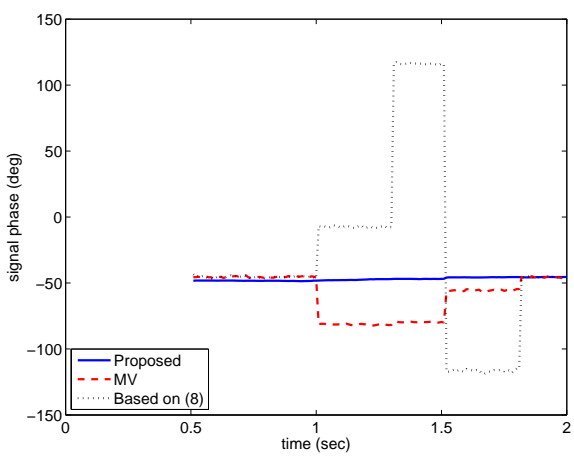

Fig. 2. Phase of the output GPS signal.

TABLE I - Parameters used in the simulations

\begin{tabular}{l|l} 
Array type: & 4-element square array \\
Inter-element spacing: & 0.4 wavelength \\
GPS PRN code: & $12 \mathrm{~dB}$ \\
GPS carrier to noise ratio: & $-10 \mathrm{~dB}$ \\
GPS Azimuth angle: & $45 \mathrm{deg}$ \\
GPS Elevation angle: & $45 \mathrm{deg}$ \\
CW Interference to noise ratio: & $55 \mathrm{~dB}$ \\
CW Azimuth angle: & $200 \mathrm{deg}$ \\
CW Elevation angle: & $60 \mathrm{deg}$ \\
BB Interference to noise ratio: & $37 \mathrm{~dB}$ \\
BB Azimuth angle: & $310 \mathrm{deg}$ \\
BB Elevation angle: & $75 \mathrm{deg}$ \\
\hline
\end{tabular}

